\title{
Pathogenesis of HIV Neurological Disease
}

National Cancer Institute

\section{Source}

National Cancer Institute. Pathogenesis of HIV Neurological Disease. NCI Thesaurus. Code $\mathrm{C18924.}$

The pathogenic mechanisms involved in HIV-associated neurological diseases and neurobehavioral dysfunction. 